\title{
Swirl Motion in a Cylindrical Bath Agitated by Top Lance Gas Injection
}

\author{
Jinlong WANG ${ }^{1,2,3)}$ Hirofumi OOYABU, ${ }^{2)}$ Fuming WANG ${ }^{1)}$ and Manabu IGUCHI ${ }^{2)}$ \\ 1) School of Metallurgical and Ecological Engineering, University of Science and Technology Beijing, Beijing, 100083 China. \\ E-mail: wangjlong78@126.com, wangfuming@metall.ustb.edu.cn 2) Graduate School of Engineering, Hokkaido \\ University, Sapporo, Hokkaido, 060-8628 Japan. E-mail: yabu@eng.hokudai.ac.jp, gaku@eng.hokudai.ac.jp \\ 3) School of Food Science and Engineering, Inner Mongolia Agricultural University, Huhehaote, 010018 China.
}

(Received on December 17, 2010; accepted on April 25, 2011)

\begin{abstract}
Researches on top lance gas injection in a cylindrical bath are carried out based on water model experiments. The penetration depth of bubbles and a swirl motion are investigated because of their practical importance. Empirical equations are proposed for the penetration depth as a function of a modified Froude number. A bath surface oscillation map is drawn in order to identify the occurrence condition of the swirl motion, and then empirical equations are proposed for describing the occurrence condition of the swirl motion. The characteristics of the swirl motion are also analyzed, and empirical equations are proposed to predict starting time, period, amplitude, and damping time of the swirl motion.
\end{abstract}

KEY WORDS: gas injection; top lance; swirl motion; penetration depth.

\section{Introduction}

Effective agitation of a molten metal bath is required in the steelmaking industry to decrease $\mathrm{CO}_{2}$ emission. Gas injection is considered to be the most economical and efficient way in all agitation methods. ${ }^{1,2)}$ Conventionally, there are three types of gas injection techniques: bottom gas injection, top gas injection, and combined bottom and top gas injection. In all kinds of gas injection modes, a swirl motion of the bath is of interest in applications in the steelmaking industry because the swirl motion is promising to enhance mixing and metallurgical reactions, although it causes severe erosion of the refractory and the vessel oscillations. ${ }^{3)}$ Therefore, it is necessary to study the occurrence condition and the basic characteristics of the swirl motion.

The characteristics of a swirl motion caused by bottom gas injection have been investigated by many researchers, and, as a result, much information is available. ${ }^{4-8)}$ Iguchi et al. proposed empirical equations for the occurrence condition of the swirl motion, and starting time, period, amplitude, and damping time of the swirl motion induced by bottom gas injection. ${ }^{5,7,8)}$ These equations could satisfactorily predict the related contents. Fundamental studies on the mass transfer between molten metal and slag and mixing time in a bath agitated by gas injection through a submerged top lance have been extensively made for the improvement of conventional steelmaking processes. $^{9-11)}$ Investigations on occurrence condition and the characteristics of a swirl motion caused by top gas injection are not enough yet.

Particular attentions were paid to the penetration depth of bubbles and a swirl motion induced by top lance gas injection in this study. Some empirical equations were proposed for predicting the penetration depth of bubbles, the occurrence condition of the swirl motion, and the starting time, period, amplitude, and damping time of the swirl motion.

\section{Experiment}

Figure 1 shows a schematic of the experimental apparatus. The vessel was made of transparent acrylic resin. The height of the cylindrical vessel, $H$, was $0.400 \mathrm{~m}$, and the diameter, $D$, was $0.200 \mathrm{~m}$. Air was injected into the bath from a top lance. The bath depth, $H_{\mathrm{L}}$, was kept at $0.300 \mathrm{~m}$. The inner and outer diameters of the lance, $d_{\mathrm{ni}}$ and $d_{\mathrm{no}}$, were $3.7 \times 10^{-3} \mathrm{~m}$ and $5.1 \times 10^{-3} \mathrm{~m}$, respectively. The lance was placed on the centerline of the vessel. The ratio of the immersion depth of the lance, $H_{\text {in }}$, to the vessel diameter, $D$, was varied from 0.10 to 1.00 . The air flow rate, $Q_{\mathrm{g}}$, was adjusted from $1 \times 10^{-5} \mathrm{~m}^{3} / \mathrm{s}$ to $1.9 \times 10^{-3} \mathrm{~m}^{3} / \mathrm{s}$ by a mass flow

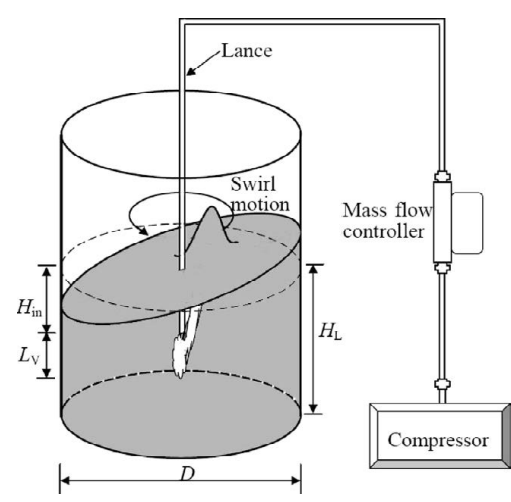

Fig. 1. Schematic of experimental apparatus. 
controller. The behavior of the bath surface was observed by visual inspection and with a digital video camera. The measurements were repeated three times under every experimental condition. Here, $L_{\mathrm{V}}$ denotes the penetration depth of bubbles which is the vertical distance from the lance exit to the lower edge of the main bubble despersion region below the lance.

\section{Results and Discussion}

\subsection{Penetration Depth of Bubbles}

The relation between the penetration depth of bubbles, $L_{\mathrm{V}}$, and the modified Froude number, $F r_{\mathrm{m}}$, are shown in Fig. 2. The modified Froude number is a dimensionless parameter representing the ratio of the inertial force of injected gas to the buoyancy force acting on a bubble generated at the lance exit. $^{10)}$

$$
F r_{\mathrm{m}}=\left(\rho_{\mathrm{g}} Q_{\mathrm{g}}^{2}\right) /\left(\rho_{\mathrm{L}} g d_{\mathrm{ni}}^{5}\right)
$$

where $g$ is the acceleration due to gravity, and $\rho_{\mathrm{g}}$ and $\rho_{\mathrm{L}}$ are the densities of gas and liquid, respectively. The three lines in Fig. 2 show the following empirical equations.

$$
\begin{gathered}
L_{\mathrm{V}} / d_{\mathrm{ni}}=1.84 F r_{\mathrm{m}}^{0.11} \quad F r_{\mathrm{m}} \leq 0.5 \ldots \ldots . . \\
L_{\mathrm{V}} / d_{\mathrm{ni}}=2.43 F r_{\mathrm{m}}^{1 / 2} \quad 0.5<F r_{\mathrm{m}} \leq 4.32 \ldots \\
L_{\mathrm{V}} / d_{\mathrm{ni}}=3.09 F r_{\mathrm{m}}^{1 / 3} \quad 4.32<F r_{\mathrm{m}} \ldots \ldots \ldots . . .
\end{gathered}
$$

The inertial force of gas injected from the top lance in the low gas flow rate range $\left(F r_{\mathrm{m}} \leq 0.5\right)$ is not strong and, hence, the penetration depth of bubbles, $L_{\mathrm{V}}$, in this range is closely associated with the diameter of a bubble generated at the exit of the top lance. Meanwhile, in the high gas flow rate range $\left(4.32<F r_{\mathrm{m}}\right)$ the penetration depth of bubbles is mainly governed by the inertial force of gas. The gas flow rate range from $F r_{\mathrm{m}}$ of 0.5 to 4.32 is the transitional range. It can be seen in Fig. 2 that the measured values are satisfactorily predicted by Eqs. (2) through (4).

In the previous paper, we derived the following empirical equation based on an electroresistivity probe measurement. ${ }^{10)}$

$$
L_{\mathrm{V}} / d_{\mathrm{ni}}=4.10 F r_{\mathrm{m}}^{1 / 3} \quad 2<F r_{\mathrm{m}}<6 \times 10^{3} .
$$

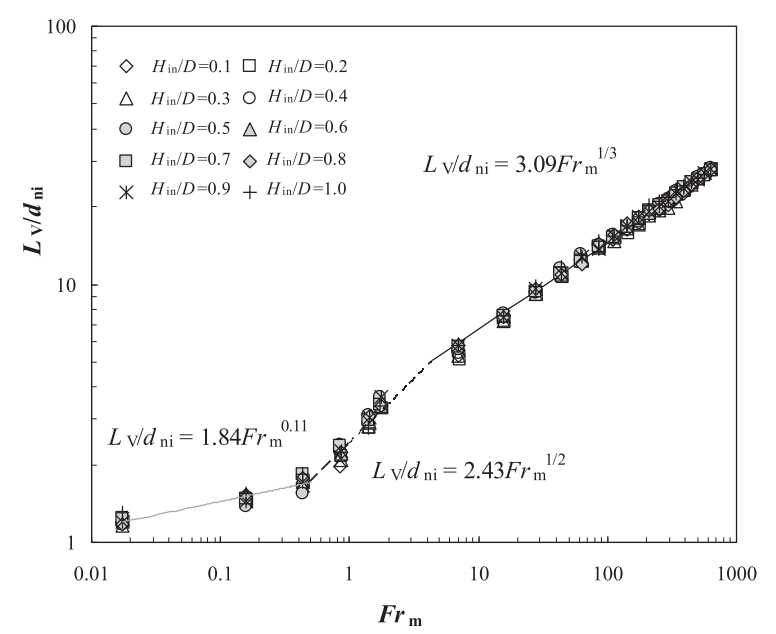

Fig. 2. Relation between penetration depth of bubbles and modified Froude number.
The penetration depth of bubbles, $L_{\mathrm{V}}$, was defined as the distance from the lance exit to the vertical position at which the gas holdup, $\alpha$, vanishes to zero. The penetration depth of bubbles, $L_{\mathrm{V}}$, changes with time around a mean value. The previously obtained equation, Eq. (5), denotes the maximum value of the penetration depth, while the presently defined penetration depth denotes the mean value. This is the reason why the presently obtained empirical equation gives a shorter penetration depth than Eq. (5).

\subsection{Occurrence Condition of Swirl Motion}

\subsubsection{Swirl Motion of the Deep-water Wave Type}

There appear two types of swirl motions depending mainly on the aspect ratio, $H_{\text {in }} / D$ : the shallow-water wave type and the deep-water wave type. The former appears for a smaller $H_{\text {in }} / D$ and the latter for a greater $H_{\text {in }} / D$. In this study, we focus on the deep-water wave type of swirl motion because of its practical importance. An example of the deepwater wave type of swirl motion is shown in Fig. 3. The swirl motion is caused by bath surface oscillations associated with the impingement of the bubbling jet onto the bath surface. The liquid around the swirling jet chases after the bubbling jet, while that away from the swirling jet rotates in the opposite direction in order to satisfy the conservation of angular momentum.

\subsubsection{Occurrence Region of Swirl Motion}

The bath surface oscillations in this study were classified into three types, as shown in Fig. 4. The lines indicated by (I), (II), (III), and (IV) denote the boundaries of the occurrence region of the swirl motion of the deep-water type for bottom gas injection. ${ }^{8)}$ The empirical equations are expressed as follow:

Sub-boundary (I):

$$
\begin{gathered}
H_{\mathrm{in}} / D=0.19 F r_{\mathrm{mD}}^{-1 / 20} \\
F r_{\mathrm{mD}}=Q_{g}^{2} /\left(g D^{5}\right) \ldots
\end{gathered}
$$

where $F r_{\mathrm{mD}}$ is the modified Froude number. The sub-boundary (I) is the limit between the deep-water wave and shallowwater wave type of swirl motion.

Sub-boundary (II):

$$
\begin{aligned}
H_{\text {in }} / D & =(1.0+0.3 \log \mathrm{We})^{1 / 2} \\
\mathrm{We} & =\rho_{L} Q_{g}^{2} /\left(\sigma D^{3}\right) \ldots \ldots .
\end{aligned}
$$

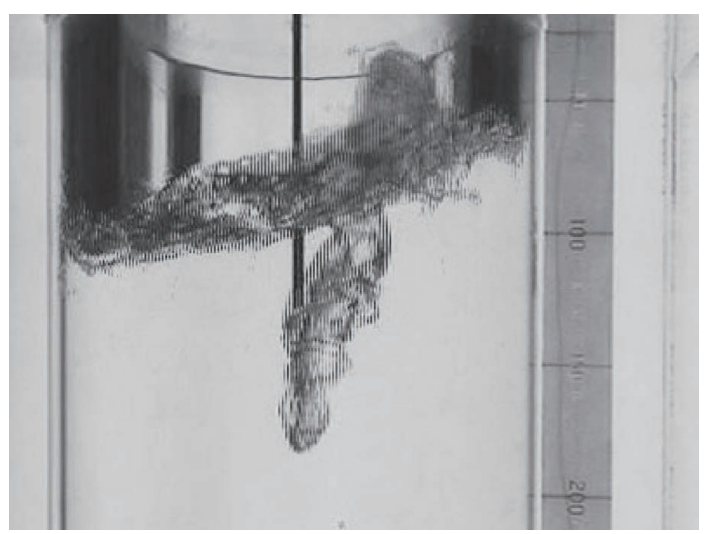

Fig. 3. Photograph of swirl motion of deep-water wave type. 


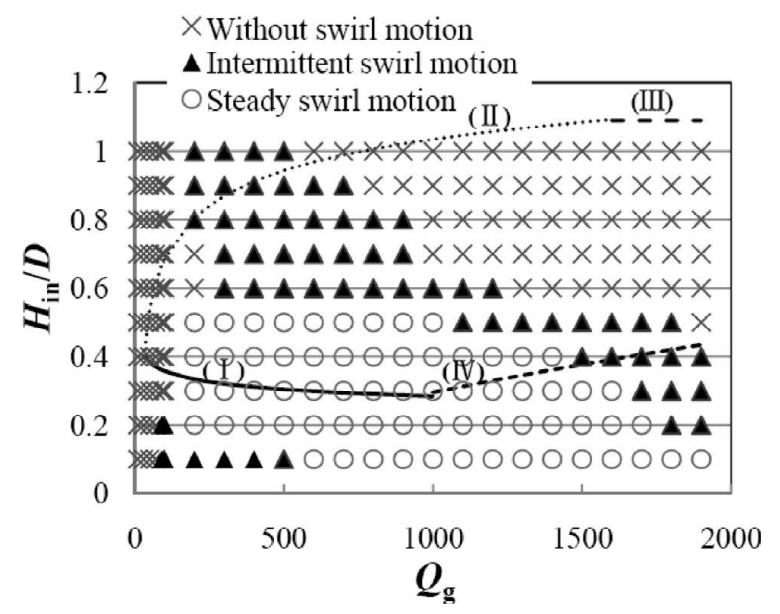

Fig. 4. Occurrence region of swirl motion of deep-water wave type.

where We is the Weber number and $\sigma$ is the surface tension. The sub-boundary (II) shows the condition that a swirl motion appears when the pressure fluctuation at the bath surface caused by impingement of a bubbling jet exceeds a certain critical value.

Sub-boundary (III):

$$
H_{\text {in }} / D=1.09
$$

The sub-boundary (III) denotes the condition that a swirl motion is suppressed as the radial displacement of the bubbling jet at the bath surface is too large.

Sub-boundary (IV):

$$
H_{\text {in }} / D=3.4\left[\frac{\rho_{\mathrm{g}} Q_{\mathrm{g}}^{2}}{\rho_{\mathrm{L}} g}\right]^{0.3} /\left(D d_{\mathrm{ni}}^{0.5}\right)
$$

The sub-boundary (IV) shows the condition that the bubbling jet penetrates too high above the bath surface and hence the swirl motion cannot occur.

The boundaries of the occurrence region of the swirl motion observed in this study cannot be approximated by the empirical equations for bottom gas injection. So it is necessary to modify the empirical equations to fit the boundaries for top gas injection.

\subsubsection{Modified Empirical Equations}

Sub-boundary (I):

Bubbles generated by top gas injection first migrated in the downward direction, and then went up. The fact means as if the nozzle exit is lowered. The identical way is used to modify the empirical equations due to a similar phenomenon with the reference. ${ }^{12)}$ Equation (6) is changed into Eq. (12).

$$
\left(H_{\mathrm{in}}+k_{1} D\right) / D=0.19 F r_{\mathrm{mD}}^{-1 / 20}
$$

where $k_{1}$ is a fitting parameter, and its value was found to be 0.2 . Accordingly, Eq. (12) reduces to

$$
\left(H_{\mathrm{in}}+0.2 D\right) / D=0.19 F r_{\mathrm{mD}}^{-1 / 20}
$$

Sub-boundary (II):

In the same way with the sub-boundary (I), Eq. (8) was modified as follows:

$$
\left(H_{\text {in }}+0.2 D\right) / D=(1.0+0.3 \log \mathrm{We})^{1 / 2}
$$

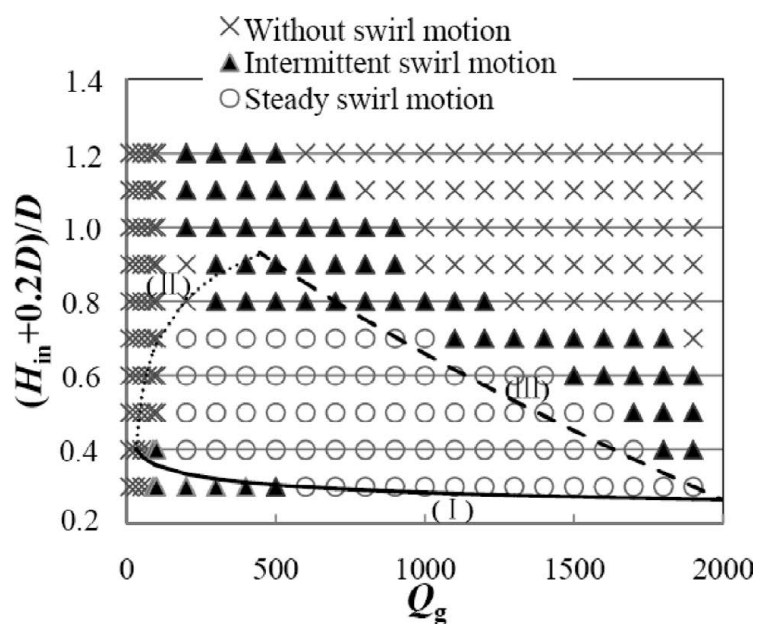

Fig. 5. Comparison of the boundaries of occurrence region of swirl motion with newly derived empirical equations.

Sub-boundary (III)

Equation (10) was derived by assuming that the swirl motion ceases when the diameter of a plume eye on the bath surface exceeds a certain value which is known to be $0.61 \mathrm{D}$ for bottom gas injection. ${ }^{8)}$ The same criterion was used for top gas injection.

$$
\begin{gathered}
D_{\text {jet }} / D=0.61 \\
D_{\text {jet }}=k_{2} L_{\mathrm{V}}+4 b_{\mathrm{u}} \\
L_{\mathrm{V}}=3.09 d_{\mathrm{ni}} F r_{\mathrm{m}}^{1 / 3} \\
b_{\mathrm{u}}=0.14 H_{\mathrm{in}} \ldots
\end{gathered}
$$

where $D_{\text {jet }}$ is the plume eye diameter, $b_{\mathrm{u}}$ is the half-value radius of water flow velocity distribution in the bubbling jet, and $k_{2}$ is a fitting parameter. Combining Eqs. (15) through (18) yields

$$
H_{\text {in }} / D=1.09-5.5 k_{2} d_{\text {ni }} F r_{\mathrm{m}}^{1 / 3} / D
$$

The fitting parameter, $k_{2}$, was found to be 1.07 . After replacing $H_{\text {in }}$ by $H_{\text {in }}+0.2 D$, Eq. (19) reduces to

$$
\left(H_{\mathrm{in}}+0.2 D\right) / D=1.09-5.90 d_{\mathrm{ni}} F r_{\mathrm{m}}^{1 / 3} / D
$$

Sub-boundary (IV):

This boundary was not observed in this study. The subboundary (IV) for bottom gas injection denotes the condition that a bubbling jet blows out of the bath surface due to strong inertial force of injected gas. As gas is injected downwards in this study, there exists no blowing out phenomenon. That is, the sub-boundary (IV) does not appear in a bath agitated by top lance gas injection.

The modified Eqs. (13), (14), and (20) were drawn in Fig. 5. It can be seen in Fig. 5 that these modified equations are in satisfactory agreement with the boundaries of the occurrence region of the swirl motion.

\subsection{The Characteristics of Swirl Motion}

3.3.1. Starting Time of Swirl Motion of Deep-water Wave Type

The starting time of swirl motion, $T_{S, S}$, is defined as the 
period from the start of gas injection into the bath to the moment at which the swirl motion attains a steady state. Figure 6 shows the experimental results of the starting time of swirl motion. The solid line denotes the values calculated from the empirical Eqs. (21) and (23) for bottom gas injection, ${ }^{5)}$ and the broken lines denote $\pm 50 \%$ deviation of Eqs. (21) and (23).

$$
\begin{gathered}
T_{S, S}(g / D)^{1 / 2}=15\left[\operatorname{Re}^{1 / 2}\left(H_{\text {in }} Q_{\mathrm{g}} / D^{7 / 2} g^{1 / 2}\right)\right]^{-1} \ldots \\
0.02<\operatorname{Re}^{1 / 2}\left(H_{\mathrm{in}} Q_{\mathrm{g}} / D^{7 / 2} g^{1 / 2}\right) \leq 0.174 \\
\operatorname{Re}=\left(Q_{\mathrm{g}}^{2} / g\right)^{2 / 5}(g / D)^{1 / 2} / \nu_{L} \ldots \ldots \ldots \ldots . \\
T_{S, S}(g / D)^{1 / 2}=36\left[\operatorname{Re}^{1 / 2}\left(H_{\mathrm{in}} Q_{\mathrm{g}} / D^{7 / 2} g^{1 / 2}\right)\right]^{-1 / 2} \ldots \ldots . . \\
0.174<\operatorname{Re}^{1 / 2}\left(H_{\mathrm{in}} Q_{\mathrm{g}} / D^{7 / 2} g^{1 / 2}\right)
\end{gathered}
$$

where Re is the Reynolds number and $v_{L}$ is the kinematic viscosity of water.

As noted, bubbles generated by top gas injection first migrated in the downward direction, and then went up. This bubble behavior is different from that for the bottom gas injection. The parameter $H_{\text {in }}$ was also modified into $\left(H_{\text {in }}+0.2 D\right)$ according to the results of the section 3.2.3. The results are shown in Fig. 7, and they are underestimated by the empirical equations. The following equation, therefore, was derived.

$$
T_{S, S}(g / D)^{1 / 2}=68.6\left\{\operatorname{Re}^{1 / 2}\left[\left(H_{\mathrm{in}}+0.2 D\right) Q_{\mathrm{g}} / D^{7 / 2} g^{1 / 2}\right]\right\}^{-0.66}
$$

Equation (24) is shown in Fig. 8. The measured values are in satisfactory agreement with the newly derived empirical equation. The swirl motion of a bath is caused by impingement of the upward-moving liquid induced by injected bubbles to the bath surface. The liquid motion is dependent on the type of gas injection. This is the reason why the starting time of swirl motion depends strongly on the gas injection type. Meanwhile, the period and amplitude of the swirl motion are not so significantly affected by the

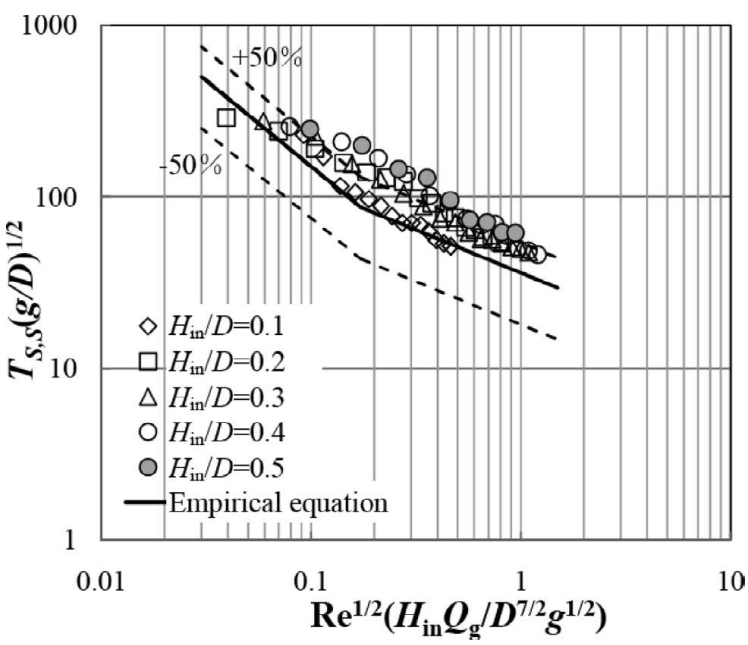

Fig. 6. Comparison of the measured values of the starting time of swirl motion with Eqs. (21) and (23). gas injection type because the swirl motion is a kind of hydro-dynamically unstable motion. Accordingly, the damping time also is not sensitive to the gas injection type.

\subsubsection{Period of Swirl Motion of Deep-water Wave Type}

The period of swirl motion, $T_{S}$, is defined as the time required for a bubbling jet to swirl around the vessel axis once. The experimental results of the period of swirl motion are shown in Fig. 9. The theoretical Eq. (25) for the rotary sloshing was chosen to predict the period of swirl motion because the swirl motion is very similar to the rotary sloshing. ${ }^{7)}$ Equation (25) was converted into Eq. (26) based on the identical reason discussed in the section 3.3.1. The results are shown in Fig. 10. By comparing Fig. 9 with Fig. 10 , it can be found that experimental results are more adequately approximated by Eq. (26) than Eq. (25).

$$
T_{S}=2 \pi\left[\left(2 g \varepsilon_{1} / D\right) \tanh \left(2 \varepsilon_{1} H_{\text {in }} / D\right)\right]^{-1 / 2}
$$

where $\varepsilon_{1}(=1.84)$ is the first zero of the Bessel function of the first kind.

$$
T_{S}=2 \pi\left\{\left(2 g \varepsilon_{1} / D\right) \tanh \left[2 \varepsilon_{1}\left(H_{\text {in }}+0.2 D\right) / D\right]\right\}^{-1 / 2}
$$

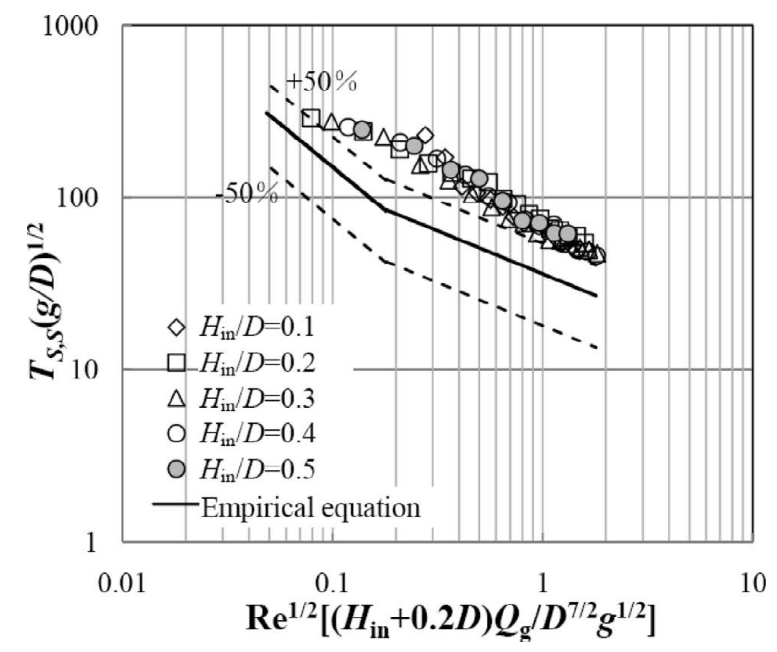

Fig. 7. Comparison of the measured values of the starting time of swirl motion with Eqs. (21) and (23) $\left(\left(H_{\text {in }}+0.2 D\right)\right.$ is substituted into Eqs. (21) and (23)).

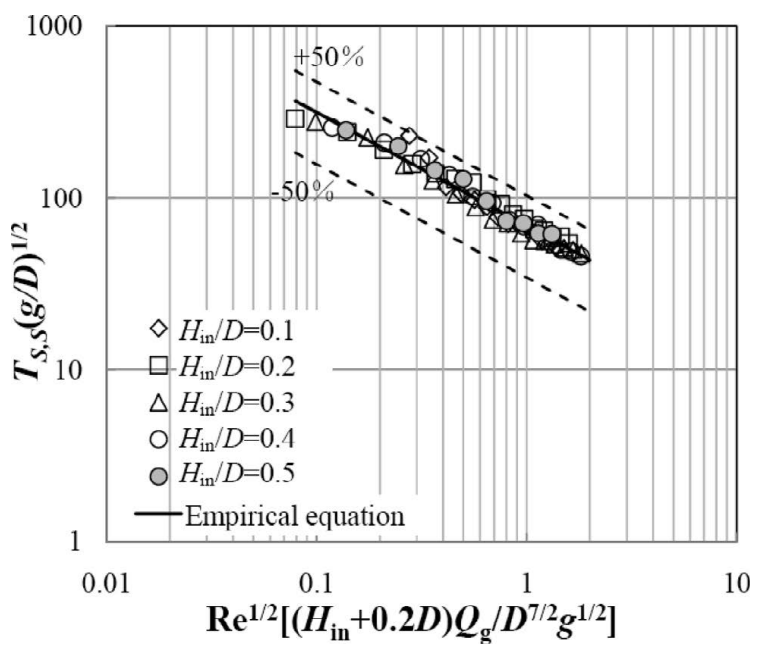

Fig. 8. Comparison of the measured values of the starting time of swirl motion with Eq. (24) 


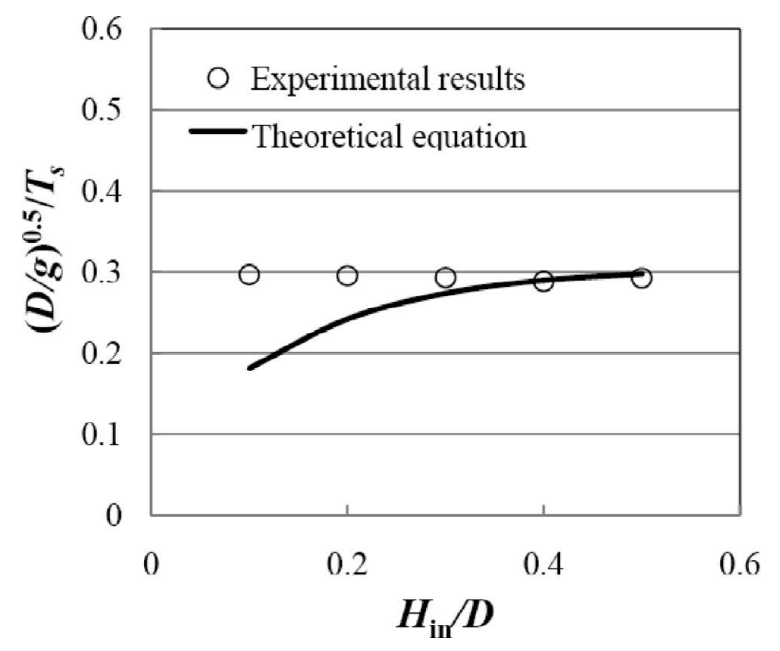

Fig. 9. Comparison of the measured values of the period of swirl motion with theoretical Eq. (25) for rotary sloshing.

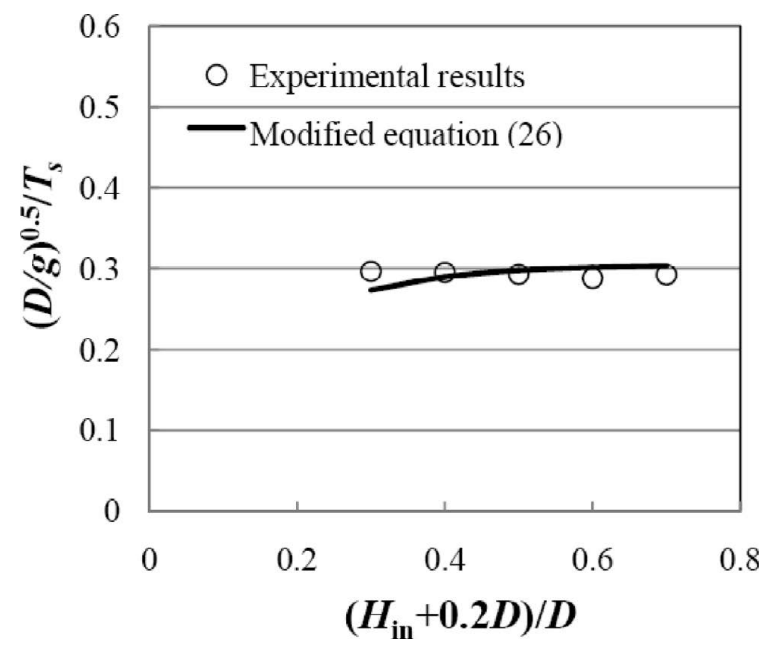

Fig. 10. Comparison of the measured values of the period of swirl motion with Eq. (26).

3.3.3. Amplitude of Swirl Motion of the Deep-water Wave Type

The amplitude of swirl motion, $A$, is defined as a half of the difference between the highest and lowest water surface positions on the side wall of the vessel. The empirical Eq. (27) derived for bottom gas injection was compared with the data of the amplitude of swirl motion in Fig. 11. ${ }^{5)}$ In the same manner as above, Eq. (27) was converted into Eq. (28) for top gas injection. The measured values of $A$ are shown in Fig. 12. It can be found from Figs. 11 and 12 that Eq. (28) is more satisfactory than Eq. (27).

$$
\begin{gathered}
A / D=0.224\left[\operatorname{Re}^{1 / 2}\left(H_{\text {in }} Q_{\mathrm{g}} / D^{7 / 2} g^{1 / 2}\right)\right]^{0.30} \ldots . . \\
A / D=0.224\left\{\operatorname{Re}^{1 / 2}\left[\left(H_{\text {in }}+0.2 D\right) Q_{\mathrm{g}} / D^{7 / 2} g^{1 / 2}\right]\right\}^{0.30} \ldots
\end{gathered}
$$

\subsubsection{Damping Time of Swirl Motion of Deep-water} Wave Type

The damping time of swirl motion, $T_{S, d}$, is defined as the period from the stoppage of gas injection to the moment at which the amplitude of swirl motion reduced to $0.5 \mathrm{~mm}$. The experimental results of the damping time of swirl motion cannot be predicted by the empirical Eq. (29)

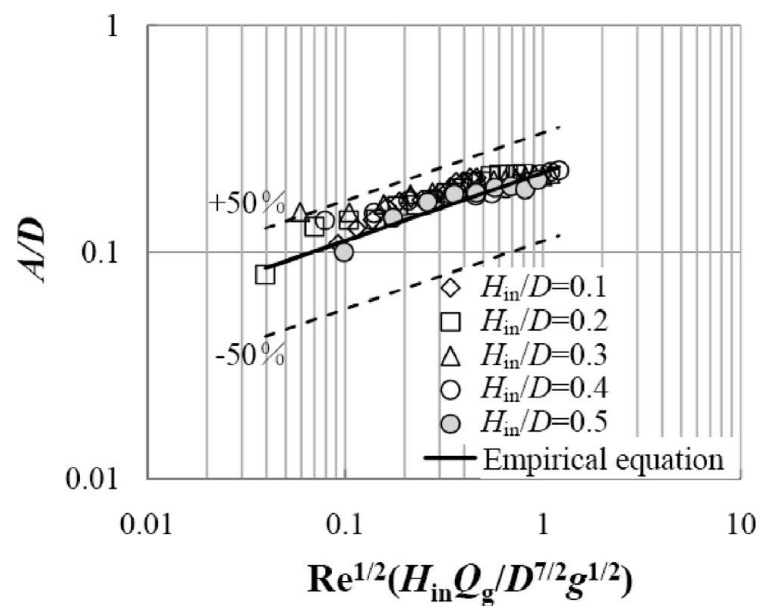

Fig. 11. Comparison of the measured values of the amplitude of swirl motion with Eq. (27).

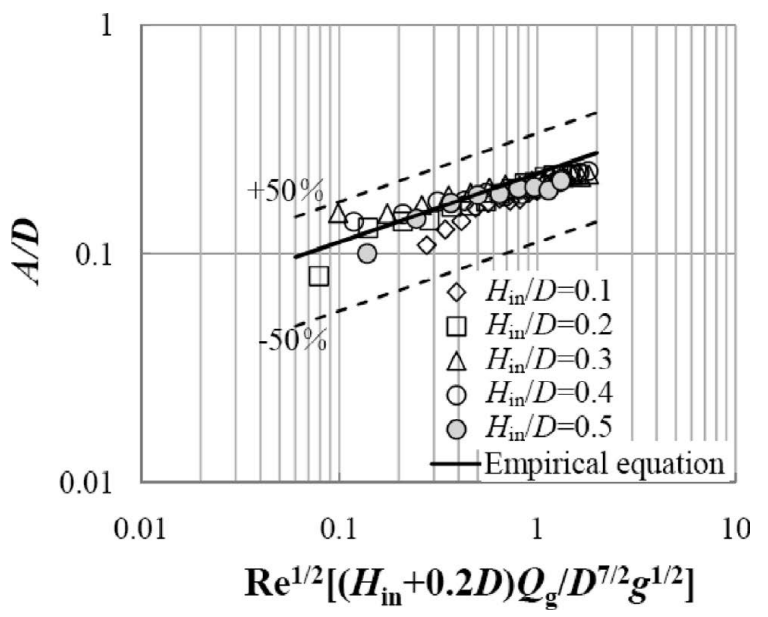

Fig. 12. Comparison of the measured values of the amplitude of swirl motion with Eq. (28).

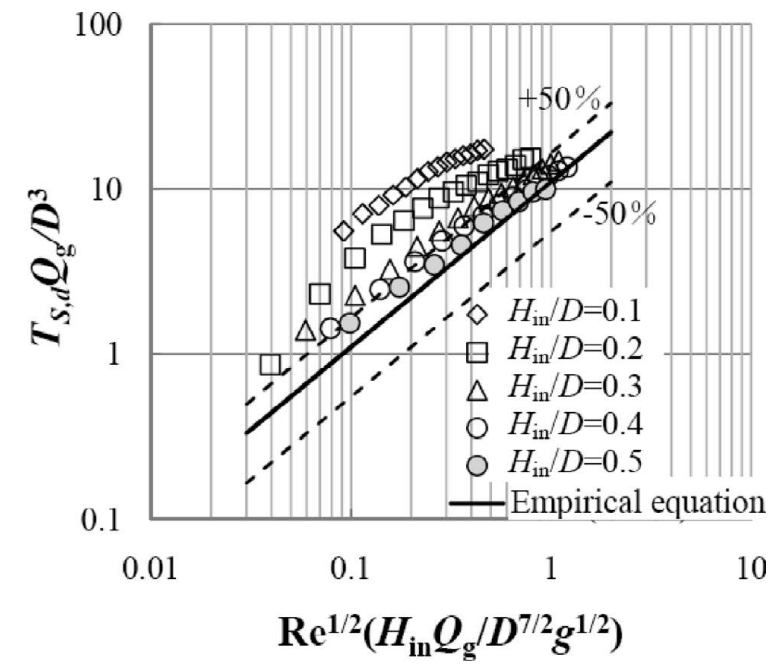

Fig. 13. Comparison of the measured values of the damping time of swirl motion with Eq. (29).

derived for bottom gas injection, as can be seen in Fig. 13. ${ }^{5)}$ Therefore, Eq. (29) was changed into Eq. (30) according to the same way as mentioned above. A comparsion of the measured values of $T_{S, d}$ with Eq. (30) is shown in Fig. 14. It can be seen from Fig. 14 that Eq. (30) is more adequate 


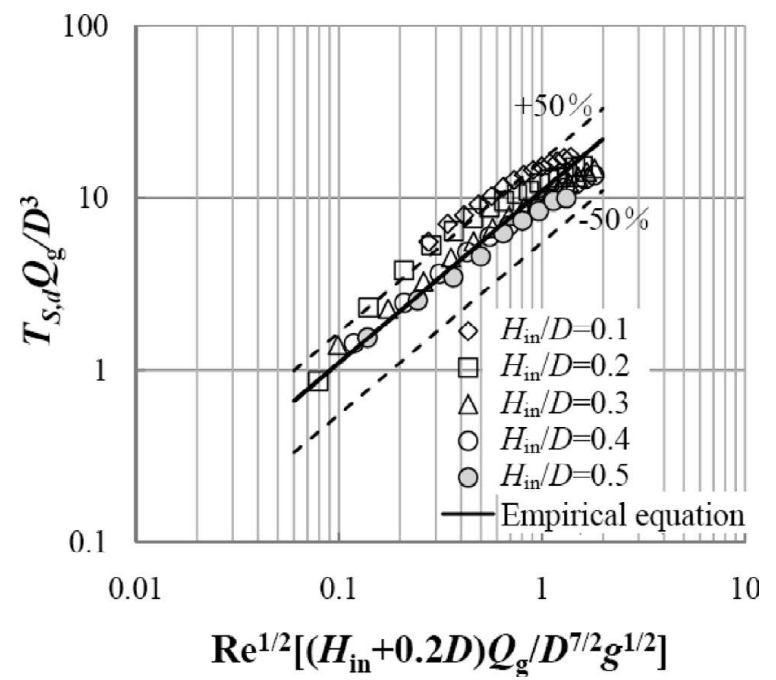

Fig. 14. Comparison of the measured values of the damping time of swirl motion with Eq. (30).

than Eq. (29).

$$
\begin{array}{r}
T_{S, d} Q_{g} / D^{3}=11 \operatorname{Re}^{1 / 2}\left(H_{\mathrm{in}} Q_{\mathrm{g}} / D^{7 / 2} g^{1 / 2}\right) \ldots \\
T_{S, d} Q_{g} / D^{3}=11 \operatorname{Re}^{1 / 2}\left[\left(H_{\mathrm{in}}+0.2 D\right) Q_{\mathrm{g}} / D^{7 / 2} g^{1 / 2}\right]
\end{array}
$$

\section{Conclusions}

Experiments on the deep-water wave type of swirl motion caused by top lance gas injection in a cylindrical bath were carried out by using a water model. The following empirical equations were proposed.

\subsection{The Vertical Penetraion Depth of Bubbles for Top Lance Gas Injection}

$$
\begin{gathered}
L_{\mathrm{V}} / d_{\mathrm{ni}}=1.84 F r_{\mathrm{m}}^{0.11} \quad F r_{\mathrm{m}} \leq 0.5 \ldots \ldots \\
L_{\mathrm{V}} / d_{\mathrm{ni}}=2.43 F r_{\mathrm{m}}^{1 / 2} \quad 0.5<F r_{\mathrm{m}} \leq 4.32 \\
L_{\mathrm{V}} / d_{\mathrm{ni}}=3.09 F r_{\mathrm{m}}^{1 / 3} \quad 4.32<F r_{\mathrm{m}} \ldots .
\end{gathered}
$$

\subsection{The Boundary of the Occurrence Region of the Swirl Motion of the Deep-water Wave Type.}

The boundary was devided into the following three subboundaries.
Sub-boundary (I):

$$
\left(H_{\text {in }}+0.2 D\right) / D=0.19 F r_{\mathrm{mD}}^{-1 / 20}
$$

Sub-boundary (II):

$$
\left(H_{\text {in }}+0.2 D\right) / D=(1.0+0.3 \log \mathrm{We})^{1 / 2}
$$

Sub-boundary (III):

$$
\left(H_{\mathrm{in}}+0.2 D\right) / D=1.09-5.90 d_{\mathrm{ni}} F r_{\mathrm{m}}^{1 / 3} / D
$$

\subsection{The Characteristics of Swirl Motion}

(1) Starting time:

$$
T_{S, S}(g / D)^{1 / 2}=68.6\left\{\operatorname{Re}^{1 / 2}\left[\left(H_{\text {in }}+0.2 D\right) Q_{\mathrm{g}} / D^{7 / 2} g^{1 / 2}\right]\right\}^{-0.66}
$$

(2) Period:

$$
T_{S}=2 \pi\left\{\left(2 g \varepsilon_{1} / D\right) \tanh \left[2 \varepsilon_{1}\left(H_{\text {in }}+0.2 D\right) / D\right]\right\}^{-1 / 2}
$$

(3) Amplitude:

$$
A / D=0.224\left\{\operatorname{Re}^{1 / 2}\left[\left(H_{\text {in }}+0.2 D\right) Q_{\mathrm{g}} / D^{7 / 2} g^{1 / 2}\right]\right\}^{0.30} \ldots
$$

(4) Damping time:

$$
T_{S, d} Q_{g} / D^{3}=11 \operatorname{Re}^{1 / 2}\left[\left(H_{\text {in }}+0.2 D\right) Q_{\mathrm{g}} / D^{7 / 2} g^{1 / 2}\right]
$$

\section{REFERENCES}

1) M. Iguchi, T. Uemura, F. Yamamoto and Z. Morita: Jpn. J. Multiphase Flow, 6 (1992), 54.

2) M. Iguchi, H. Ueda and T. Uemura: Int. J. Multiphase Flow, 21 (1995), 861.

3) M. J. Doby, A. F. Nowakowski, E. Nowak and T. Dyakowski: Miner. Eng., 20 (2007), 361.

4) M. P. Schwartz: Chem. Eng. Sci., 45 (1990), 1765.

5) M. Iguchi, Y. Itoh and Z. Morita: Testsu-to-Hagané, 80 (1994), 189.

6) T. Fujikawa, Y. Sasaki, M. Fukue and M. Iguchi: J. JSEM, 7 (2007), 343.

7) M. Iguchi, S. Hosohara, T. Koga, R. Yamaguchi and Z. Morita: ISIJ Int., 33 (1993), 1037.

8) M. Iguchi, D. Iguchi, Y. Sasaki, T. Kumagai and S. Yokoya: ISIJ Int., 44 (2004), 1623.

9) O. J. Ilegbusi, M. Iguchi and W. Wahnsiedler: Mathematical and Physical Modeling of Materials Processing Operations, Chapman \& Hall/CRC, Florida, (2000), 442.

10) M. Iguchi, T. Uemura, H. Yamaguchi, T. Kuranaga and Z. Morita: ISIJ Int., 34 (1994), 973.

11) Y. Fukunaka, M.-F. Jiang, T. Yamamoto, Z. Asaki and Y. Kondo: Metall. Trans., 20B (1989), 5.

12) Y. Nasukawa, T. Fujikawa, R. Tsujino and M. Iguchi: Mater. Trans., 51 (2010), 1560 